\title{
Transmission dynamics model of Tuberculosis with optimal control strategies in Haramaya district, Ethiopia
}

\author{
Doyo Kereyu ${ }^{1}$ and Seleshi Demie ${ }^{1 *}$ (E)
}

"Correspondence:

seleshidemie@gmail.com

${ }^{1}$ Department of Mathematics,

College of Natural and

Computational Sciences, Haramaya

University, Haramaya, Ethiopia

\section{Springer}

\begin{abstract}
In this study, we use a compartmental nonlinear deterministic mathematical model to investigate the effect of different optimal control strategies in controlling Tuberculosis (TB) disease transmission in the community. We employ stability theory of differential equations to investigate the qualitative behavior of the model by obtaining the basic reproduction number and determining the local stability conditions for the disease-free and endemic equilibria. We consider three control strategies representing distancing, case finding, and treatment efforts and numerically compare the levels of exposed and infectious populations with and without control strategies. The results suggest that combination of all controls is the best strategy to eradicate TB disease from the community at an optimal level with minimum cost of interventions.
\end{abstract}

Keywords: Tuberculosis; Dynamic model; Ethiopia; Optimal control; Pontryagin maximum principle

\section{Introduction}

TB is a chronic infection of the bacteria caused by Mycobacterium TB, which was discovered by Koch in 1882. Different scholars classify TB in various ways based on the symptoms and treatments of the disease. The medical community divides TB into two categories, pulmonary (smear-positive and smear-negative) and extra-pulmonary. Pulmonary TB is a common form of TB that affects the lungs, whereas extra-pulmonary TB affects other parts of the body such as pleura, lymph nodes, back, joints, urinary tract of the genitor, nervous system, and abdomen [14]. A susceptible person is infected with TB when he or she inhales the TB germs that are released into the air while the infectious person with pulmonary TB disease coughs, sneezes, cries, or sings [6, 14]. The first stage of infection is the latency period. At this stage an individual does not exhibit any symptoms of the disease and does not infect others. Such individual is said to be infected with latent TB. The second stage is the time of active TB infection when a person begins to display symptoms such as general weakness or fatigue, fever, weight loss, lack of appetite, and night sweating and can also infect others [19].

TB is an ancient disease, which caused more suffering and death than any other infectious disease and remains a significant global health issue. Even today, after advanced

(c) The Author(s) 2021. This article is licensed under a Creative Commons Attribution 4.0 International License, which permits use sharing, adaptation, distribution and reproduction in any medium or format, as long as you give appropriate credit to the original author(s) and the source, provide a link to the Creative Commons licence, and indicate if changes were made. The images or other third party material in this article are included in the article's Creative Commons licence, unless indicated otherwise in a credit line to the material. If material is not included in the article's Creative Commons licence and your intended use is not permitted by statutory regulation or exceeds the permitted use, you will need to obtain permission directly from the copyright holder. To view a copy of this licence, visit http://creativecommons.org/licenses/by/4.0/. 
screening, diagnosis, and treatment methods have been established, one third of the world's population has been exposed and is infected with TB [18]. Although TB cases are currently reducing in many developed countries, they are rising in Africa, Eastern parts of Europe, and Asia [17]. The government of Ethiopia, in collaboration with development partners, bilateral and multilateral organizations, and wider community, is working together to combat this deadly disease to meet the sustainable development targets by 2030 and end TB by 2035 . However, according to the World Health Organization (WHO) report 2019 [23], Ethiopia is among 30 high TB and TB/HIV burden countries in which 70\% of notified cases are within the age group of $15-54$ years. This indicates that TB is affecting mostly the productive working group so that it can bring back the economy of the country which demands extensive work to decide the right control strategies.

Mathematical modeling of infectious disease is used as a powerful tool for studying the dynamics of disease transmission and the effect of different intervention strategies to inform public health policy makers on the implementation of effective intervention programs to combat infections. Several researchers have built different mathematical models to investigate the dynamics of TB disease transmission and optimal control strategies; for example, see $[5,15,16,20,21,24,25]$ and some references therein. Gao and Huang [12] analyzed TB model that incorporates vaccination of susceptible individuals, identification for treatment of latently infected individuals, and treatment of individuals with active TB. Their analysis showed that the combined implementation of three controls is most effective and less expensive among different strategies. In [2] an optimal TB control model was analyzed by considering the case detection of TB infections as optimal control parameter. Investigating the effectiveness of optimal control by comparing the levels of exposed and infectious populations with and without optimal control, the authors suggested that the optimal control approach would deliver better results in terms of reducing the number of infectives. Abouelkheir et al. [1] proposed a mathematical model for the optimal control strategy of a drug-resistant TB by considering a treatment program. The numerical simulations of their study showed that the implementation of a treatment as a control has a positive impact on the reduction of infectious individuals. Whang et al. [21] developed a dynamic TB transmission model in South Korea using a modified Susceptible-ExposedInfectious-Recovered model with the time-dependent parameters and proposed optimal treatment strategies. Using the data of active-TB incidence obtained from 1970 to 2009, they estimated the parameters by the least-squares curve fitting. They considered three control mechanisms (distancing, case finding, and case holding efforts) and used optimal control programs to minimize the number of exposed and infectious individuals and the cost of implementing the control treatment. The results showed that the most effective control factor for preventing TB transmission is a distancing control. All of the above studies showed substantial results for TB disease transmission dynamics by taking situations in different countries into consideration. In this paper, we propose a dynamic model for TB disease transmission in Hramaya district, Ethiopia. The model is a modified and extended version of the model presented in [9] with optimal control strategies for the control of the TB disease.

The rest of the paper is organized as follows. In Sect. 2, we describe the basic model formulation. In Sect. 3, we present qualitative analysis of the basic model. In Sect. 4, we formulate and analytically study an optimal control problem using Pontryagin's maximum 


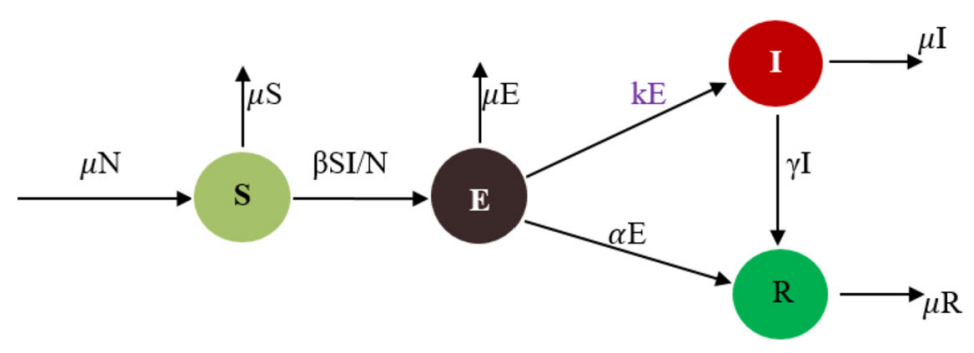

Figure 1 Model flow diagram

principle. In Sect. 5, we present the numerical results and discussions. Finally, Sect. 6 concludes the paper.

\section{Model description and formulation}

We formulate a mathematical model that describes the dynamics of TB infection in a population. The model divides individuals in the population under study into four compartments according to their epidemiological status. These are the susceptible individuals $(S)$, who are not yet infected by the TB bacterium but with a possibility to be infected; the exposed individuals $(E)$, who recently infected but are not infectious; the infectious individuals $(I)$, who have become infected with TB and are able to transmit the disease; and the recovered individuals $(R)$, who have recovered from the disease. We assume that the total population $N$ remains constant (that is, natural birth rate and death rate are equal, and there is no emigration or immigration) and there are no TB disease-related deaths. So, the natural birth and death rate is $\mu$. We also assume that an individual is firstly exposed to the disease through contacts with infectious individuals and does not become infectious instantly. That is, after the initial infection, an individual stays in a latent period for some time and then either becomes infectious (by developing the disease) or recovered (when immunological defense kills the inhaled bacilli). Hence the rate at which people become susceptible starts with rate $\mu N$. From that rate, the normal death rate of people that have not yet been exposed to $\mathrm{TB}, \mu S$, and the rate at which susceptibles by meeting with infectious become exposed, $\beta S I / N$, are subtracted. From those exposed people, those who die, $\mu E$, those who become infectious after being exposed, $\mathrm{kE}$, and those who become recovered, $\alpha E$, are reduced. To describe the rate at which people in the population become infectious, from $\mathrm{kE}$, the percentage of death among infectious, $\mu I$, and the percentage of recovery from the TB infectious, $\gamma I$, are subtracted. The last equation describes the rate at which people in the population recover from a disease; that is, individuals who do not progress to TB infectious $\alpha E$ are moved from class $E$ to the recovered class $R$, and the recovered individuals $\gamma I$ are also moved from class $I$ to $R$. From that amount, the percentage of death among recovered people $\mu R$ is subtracted. This model description is shown in Fig. 1. 
Based on this model flow diagram, the dynamics of the model is given by the following system of differential equations:

$$
\left\{\begin{array}{l}
\frac{d S}{d t}=\mu N-\mu S-\frac{\beta S I}{N}, \\
\frac{d E}{d t}=\frac{\beta S I}{N}-(\mu+k+\alpha) E, \\
\frac{d I}{d t}=k E-(\mu+\gamma) I, \\
\frac{d R}{d t}=\alpha E+\gamma I-\mu R,
\end{array}\right.
$$

with initial conditions

$$
S(0)=S_{0} \geq 0, \quad E(0)=E_{0} \geq 0, \quad I(0)=I_{0} \geq 0 \quad \text { and } \quad R(0)=R_{0} \geq 0 .
$$

The total population $N(t)=N$ at time $\mathrm{t}$ is the sum of the populations in the compartments $S, E, I$, and $R$. Since the variable $R$ does not appear in the first three equations of model (1), it is possible to examine model (1) by studying the subsystem

$$
\left\{\begin{array}{l}
\frac{d S}{d t}=\mu N-\mu S-\frac{\beta S I}{N}, \\
\frac{d E}{d t}=\frac{\beta S I}{N}-(\mu+k+\alpha) E, \\
\frac{d I}{d t}=k E-(\mu+\gamma) I,
\end{array}\right.
$$

and determining $R$ from $R=N-(S+E+I)$ or $\frac{d R}{d t}=\alpha E+\gamma I-\mu R$.

\section{Model analysis}

Since model (1) tracks human populations, all parameters associated with it are nonnegative.

\subsection{Invariant region}

From model (1) the addition of all the equations gives

$$
\frac{d N}{d t}=\frac{d S}{d t}+\frac{d E}{d t}+\frac{d I}{d t}+\frac{d R}{d t}=0
$$

which indicates that the total population $N$ is constant. Also, since model (1) represents interaction between individuals in the human population, it is plausible to assume that all the state variables of the model are nonnegative for all $t \geq 0$ (see the proof of Theorem 3.1). Further, it can be shown that the region

$$
\Omega=\left\{(S, E, I, R) \in \mathbb{R}_{+}^{4}: S+E+I+R \leq N\right\}
$$

is positively invariant. Thus the solution of model (1) with initial conditions in $\Omega$ remains there for $t \geq 0$. Furthermore, the usual existence, uniqueness, and continuation results hold in $\Omega$ for the equations of model (1) so that model (1) is well posed both mathematically and epidemiologically. So it is sufficient to study the dynamics of the basic model (1) in $\Omega$. 


\subsection{Positivity of the solutions}

Theorem 3.1 The solutions of model (1) with positive initial data remain positive for all times $t \geq 0$.

Proof Given that the initial data $S_{0}, E_{0}, I_{0}, R_{0}$ are nonnegative, it is clear from the first equation of model (1) that

$$
\frac{d S}{d t}=\mu N-\mu S-\frac{\beta S I}{N} \geq-\left(\mu+\frac{\beta I}{N}\right) S,
$$

from which we have

$$
\frac{d S}{S} \geq-\left(\mu+\frac{\beta I}{N}\right) d t .
$$

Integrating both sides of this inequality and applying the technique of separation of variables along with initial condition yield

$$
S(t) \geq S_{0} e^{-\left(\mu+\frac{\beta I}{N}\right) t} .
$$

Also from the second equation of model (1) we have

$$
\frac{d E}{d t}=\frac{\beta S I}{N}-(\mu+k+\alpha) E \geq-(\mu+k+\alpha) E,
$$

which implies

$$
\frac{d E}{E} \geq-(\mu+k+\alpha) d t .
$$

Integrating both sides, we have

$$
\int \frac{d E}{E} \geq-\int(\mu+k+\alpha) d t .
$$

Then solving by technique of separation of variables and applying the initial condition, we get

$$
E(t) \geq E_{0} e^{-(\mu+k+\alpha) t} .
$$

Applying similar steps to the third and fourth equations of model (1), we obtain, respectively,

$$
I(t) \geq I_{0} e^{-(\mu+\gamma) t}
$$

and

$$
R(t) \geq R_{0} e^{-\mu t} .
$$

This completes the proof of the theorem. 
Therefore the solution of model (1) is positive. Since studying model (1) is similar to studying the reduced model (3), we focus the following discussions on model (3).

\subsection{Equilibria and the basic reproduction number}

The system of model (3) has two nonnegative equilibrium points called the disease-free equilibrium (DFE), where $E=I=0$, and endemic equilibrium (EE), where $E \neq 0$ and $I \neq 0$. To obtain the DFE and EE points, the right-hand side of model (3) is set to zero and then solved for the values of $S, E$, and $I$. Then the DFE point of the respective states becomes

$$
E_{1}=(N, 0,0)
$$

and the EE point becomes

$$
E_{2}=\left(\frac{N}{R_{0}}, \frac{\mu N(\mu+\gamma)}{\beta k}\left(R_{0}-1\right), \frac{\mu N}{\beta}\left(R_{0}-1\right)\right) .
$$

To calculate the basic reproduction number $R_{0}$ of model (3), we use the method of the next generation matrix given in [10] and obtain

$$
R_{0}=\frac{\beta k}{(\mu+k+\alpha)(\mu+\gamma)} .
$$

\subsection{Local stability of the equilibria}

For the stability analysis of the DFE and EE points, the linearization matrix (Jacobian matrix) of model (3) is evaluated at each equilibrium point, and then its eigenvalues are determined by solving its characteristic equation. Based on the eigenvalues, the equilibrium points will either be stable (if all the eigenvalues of the Jacobian evaluated at the equilibrium point contain negative real parts) or unstable (if at least one of the eigenvalues of the Jacobian evaluated at the equilibrium point has a positive real part).

Theorem 3.2 The DFE of model (3) is locally asymptotically stable if $R_{0}<1$ and unstable if $R_{0}>1$.

Proof The Jacobian matrix of model (3) is

$$
J(S, E, I)=\left[\begin{array}{ccc}
-\mu-\frac{\beta I}{N} & 0 & -\frac{\beta S}{N} \\
\frac{\beta I}{N} & -(\mu+k+\alpha) & \frac{\beta S}{N} \\
0 & k & -(\mu+\gamma)
\end{array}\right] .
$$

For the DFE point $E_{1}$ of Eq. (10),

$$
J\left(E_{1}\right)=\left[\begin{array}{ccc}
-\mu & 0 & -\beta \\
0 & -(\mu+k+\alpha) & \beta \\
0 & k & -(\mu+\gamma)
\end{array}\right] \text {. }
$$

The eigenvalues of the Jacobian matrix J are the solutions of the characteristic equation

$$
\left|J\left(E_{1}\right)-\lambda I\right|=0
$$


where $I$ is the identity matrix, that is,

$$
\left|\begin{array}{ccc}
-\mu-\lambda & 0 & -\beta \\
0 & -(\mu+k+\alpha)-\lambda & \beta \\
0 & k & -(\mu+\gamma)-\lambda
\end{array}\right|=0 .
$$

This leads to the characteristic equation given by

$$
\lambda^{3}+a_{1} \lambda^{2}+a_{2} \lambda+a_{3}=0,
$$

where

$$
\begin{aligned}
& a_{1}=3 \mu+k+\alpha+\gamma, \\
& a_{2}=\mu(2 \mu+k+\alpha+\gamma)+(\mu+\gamma)(\mu+k+\alpha)\left(1-R_{0}\right), \\
& a_{3}=\mu(\mu+\gamma)(\mu+k+\alpha)\left(1-R_{0}\right) .
\end{aligned}
$$

Based on the Routh-Hurwitz stability criteria, without solving Eq. (17), we get that the sufficient and necessary conditions for stability are

$$
a_{1}>0, \quad a_{3}>0, \quad a_{1} a_{2}-a_{3}>0 .
$$

The first inequality is clearly satisfied since all model parameters are positive, and the second inequality $a_{3}>0$ holds if $R_{0}<1$. For the third condition,

$$
\begin{aligned}
a_{1} a_{2}-a_{3}= & (3 \mu+k+\alpha+\gamma)\left(\mu(2 \mu+k+\alpha+\gamma)+(\mu+\gamma)(\mu+k+\alpha)\left(1-R_{0}\right)\right) \\
& -\mu(\mu+\gamma)(\mu+k+\alpha)\left(1-R_{0}\right) \\
= & (2 \mu+k+\alpha+\gamma)\left(\mu(3 \mu+k+\alpha+\gamma)+(\mu+\gamma)(\mu+k+\alpha)\left(1-R_{0}\right)\right)>0 .
\end{aligned}
$$

Therefore by the Routh-Hurwitz criteria all eigenvalues of $J\left(E_{1}\right)$ are negative when $R_{0}<$ 1 , so that $E_{1}$ is locally asymptotically stable.

Theorem 3.3 The EE of model (3) is locally asymptotically stable if $R_{0}>1$ and unstable if $R_{0}<1$

Proof For the EE point $E_{2}$ of Eq. (11),

$$
J\left(E_{2}\right)=\left[\begin{array}{ccc}
-\mu R_{0} & 0 & \frac{-\beta}{R_{0}} \\
\mu\left(R_{0}-1\right) & -(\mu+k+\alpha) & \frac{\beta}{R_{0}} \\
0 & k & -(\mu+\gamma)
\end{array}\right] .
$$

Then its characteristic equation is given by

$$
\lambda^{3}+b_{1} \lambda^{2}+b_{2} \lambda+b_{3}=0,
$$


Table 1 Sensitivity indices of $R_{0}$ to model parameters

\begin{tabular}{ll}
\hline Parameter & Sensitivity indices \\
\hline$\mu$ & -0.0643 \\
$\beta$ & +1 \\
$\mathrm{k}$ & +0.84 \\
$\alpha$ & -0.8 \\
$\gamma$ & -0.99 \\
\hline
\end{tabular}

where

$$
\begin{aligned}
& b_{1}=\mu R_{0}+2 \mu+k+\alpha+\gamma, \\
& b_{2}=\mu(2 \mu+k+\alpha+\gamma) R_{0}, \\
& b_{3}=\mu(\mu+\gamma)(\mu+k+\alpha)\left(R_{0}-1\right) .
\end{aligned}
$$

Clearly, $b_{1}>0$, and if $R_{0}>1$, then $b_{3}>0$, and

$$
\begin{aligned}
b_{1} b_{2}-b_{3} & =\left(\mu R_{0}+2 \mu+k+\alpha+\gamma\right) \mu(2 \mu+k+\alpha+\gamma) R_{0}-\mu(\mu+\gamma)(\mu+k+\alpha)\left(R_{0}-1\right) \\
& =\mu R_{0}\left(\mu R_{0}+2 \mu+k+\alpha+\gamma\right)(2 \mu+k+\alpha+\gamma)+\mu((\mu+k+\alpha)(\mu+\gamma)-\beta k) \\
& >0 .
\end{aligned}
$$

Therefore by the Routh-Hurwitz criteria, all eigenvalues of $J\left(E_{2}\right)$ are negative when $R_{0}>$ 1 , so that $E_{2}$ is locally asymptotically stable.

\subsection{Sensitivity analysis of the model parameters}

In the study of biological dynamics the transmission dynamics of infectious disease model sensitivity analysis plays an important role. Using sensitivity analysis, the role of each parameter used in the model can be investigated, and a strategy can easily be developed to control the spread of infection in the community.

In this section, the normalized forward sensitivity index of $R_{0}$ to a model parameter, which is a ratio of the relative change in $R_{0}$ to the relative change in the parameter defined by

$$
\Upsilon_{P}^{R_{0}}=\frac{\partial R_{0}}{\partial p} \times \frac{P}{R_{0}}
$$

where p represents all the model parameters $(\mu, \beta, k, \alpha, \gamma)$, is carried out in the sense of [3]. Using (23), the sensitivity indices of $R_{0}$ with respect to the model parameters are presented in Table 1 using the values $\mu=0.015267, \beta=10, k=0.0444, \alpha=0.2325$, and $\gamma=1.25$ taken from Sect. 5 .

The positive sign of sensitivity index of $R_{0}$ to the model parameters indicates that an increase (or decrease) in the value of each of the parameter leads to increase (or decrease) in $R_{0}$, whereas the negative sign indicates that an increase (or decrease) in the value of parameter leads to a corresponding decrease (or increase) in $R_{0}$ of model (3). From Table 1 , $\beta$ and $k$ have the impact of expanding the disease in the community if their values are increased, whereas $\mu, \alpha$, and $\gamma$ have an influence of minimizing the burden of the disease in the community as their values increase. 


\section{Optimal control problem}

Optimal control is the method of determining control and state trajectories for a dynamic system over a period of time to minimize a performance index or an objective functional.

In this section, three time-dependent controls (distancing, case finding, and treatment) are incorporated to the model of Eq. (1). The distancing control $u_{1}(t)$ is the effort of preventing susceptible individuals from becoming infectious using strategies such as early detection and isolation of infectious individuals, and health education campaign (prevention of careless spitting, coughing, and sneezing). It is incorporated by adding a control term that characterizes the contact between susceptible and infectious individuals so that the rate of infection will be reduced. The case finding control $u_{2}(t)$ is the effort of identifying TB exposed individuals through screening and put under treatment to reduce number of individuals that may progress to TB infectious. The treatment control $u_{3}(t)$ is the effort of ensuring for those that are infectious treatment and monitoring in taking their drugs to reduce the number of individuals developing and dying of TB. Then, after incorporating the controls into the model described by Eq. (1), we get the following optimal control model:

$$
\left\{\begin{array}{l}
\frac{d S}{d t}=\mu N-\mu S-\left(1-u_{1}\right) \frac{\beta S I}{N} \\
\frac{d E}{d t}=\left(1-u_{1}\right) \frac{\beta S I}{N}-\left(\mu+k+\left(1+u_{2}\right) \alpha\right) E \\
\frac{d I}{d t}=k E-\left(\mu+\gamma+u_{3}\right) I \\
\frac{d R}{d t}=\left(1+u_{2}\right) \alpha E+\left(\gamma+u_{3}\right) I-\mu R
\end{array}\right.
$$

with the initial conditions of Eq. (2).

Here our purpose is finding the optimal values of $u_{1}, u_{2}$, and $u_{3}$ so that the state and control trajectories minimize the objective functional given by

$$
J\left(u_{1}, u_{2}, u_{3}\right)=\int_{0}^{t_{f}}\left(E+I+\frac{B_{1}}{2} u_{1}^{2}+\frac{B_{2}}{2} u_{2}^{2}+\frac{B_{3}}{2} u_{3}^{2}\right) d t
$$

subject to the state system (24).

In Eq. (25), $t_{f}$ is a fixed final time, whereas the coefficients $B_{1}, B_{2}$, and $B_{3}$ are positive weight constants, which balance the cost factors associated with control measures $u_{1}, u_{2}$, and $u_{3}$, respectively. The cost of each control measure is assumed to be nonlinear and take quadratic form, that is, $\frac{B_{1}}{2} u_{1}^{2}, \frac{B_{2}}{2} u_{2}^{2}$, and $\frac{B_{3}}{2} u_{3}^{2}$ are the costs of the control measure associated with $u_{1}, u_{2}$, and $u_{3}$, respectively. Thus we seek the optimal controls, $u_{1}^{*}, u_{2}^{*}, u_{3}^{*}$ satisfying

$$
J\left(u_{1}^{*}, u_{2}^{*}, u_{3}^{*}\right)=\min \left\{J\left(u_{1}, u_{2}, u_{3}\right): u_{1}, u_{2}, u_{3} \in U\right\}
$$

where $U=\left\{\left(u_{1}, u_{2}, u_{3}\right)\right.$ : each $u_{i}, i=1,2,3$ is Lebesgue measurable with $0 \leq u_{i} \leq u_{i \max }$ for $\left.0 \leq t \leq t_{f}\right\}$ is the set of acceptable controls.

\subsection{Existence of an optimal control}

Theorem 4.1 There exist optimal controls $u_{1}^{*}, u_{2}^{*}$, and $u_{3}^{*}$ and the corresponding state solutions $S^{*}, E^{*}, I^{*}$, and $R^{*}$ to Eqs. (24)-(26) that minimize $J\left(u_{1}, u_{2}, u_{3}\right)$ over $U$. 
Proof The nontrivial criteria regarding the set of admissible controls $U$ and the set of end conditions from the theorem of Fleming and Rishel [11] are described and checked below.

A. The set of all solutions to Eqs. (24)-(26) with corresponding control functions in $U$ is nonempty.

B. The state system (24) can be written as a linear function of the control variables with coefficients dependent on time and the state variables.

C. The integrand $\mathrm{L}$ of (25), $L\left(t, S, E, I, R, u_{1}, u_{2}, u_{3}\right)=E+I+\frac{B_{1}}{2} u_{1}^{2}+\frac{B_{2}}{2} u_{2}^{2}+\frac{B_{3}}{2} u_{3}^{2}$ is convex on $U$ and additionally satisfies $L\left(t, S, E, I, R, u_{1}, u_{2}, u_{3}\right) \geq d_{1}\left|\left(u_{1}, u_{2}, u_{3}\right)\right|^{\tau}-d_{2}$, where $d_{1}>0$ and $\tau>1$. To establish condition A, Picard-Lindelöf's theorem from [3, 4] is referred. If the solutions of the state equations are bounded and the state equations are Lipschitz continuous in the state variables, then there is a unique solution corresponding to every admissible control $U$. With the boundedness of the solutions of model (1) already justified, it follows that the state system is continuous and bounded. It is also equally direct to show the boundedness of the partial derivatives with respect to the state variables in the state system, which confirms that the system is Lipschitz in relation to the state variables [7]. This completes the proof of condition A. Condition B is confirmed by observing linear dependence on controls $u_{1}, u_{2}$, and $u_{3}$ of the state equations. Finally, to verify condition C, by definition from [8] any constant, linear, and quadratic functions are convex. Therefore $L\left(t, S, E, I, R, u_{1}, u_{2}, u_{3}\right)$ is convex on $U$. To prove the bound on $L$, note that by the definition of $U$ we have

$$
\begin{aligned}
& B_{3} u_{3}^{2} \leq B_{3} \quad \text { since } u_{3} \in\left[0, u_{3 \max }\right], \\
& \frac{B_{3}}{2} u_{3}^{2} \leq \frac{B_{3}}{2} \quad \Rightarrow \quad \frac{B_{3}}{2} u_{3}^{2}-\frac{B_{3}}{2} \leq 0, \\
& L\left(t, S, E, I, R, u_{1}, u_{2}, u_{3}\right)=E+I+\frac{B_{1}}{2} u_{1}^{2}+\frac{B_{2}}{2} u_{2}^{2}+\frac{B_{3}}{2} u_{3}^{2} \geq \frac{B_{1}}{2} u_{1}^{2}+\frac{B_{2}}{2} u_{2}^{2}+\frac{B_{3}}{2} u_{3}^{2}-\frac{B_{3}}{2} \\
& \Rightarrow \quad L\left(t, S, E, I, R, u_{1}, u_{2}, u_{3}\right) \geq \min \left(\frac{B_{1}}{2}, \frac{B_{2}}{2}, \frac{B_{3}}{2}\right)\left(u_{1}^{2}+u_{2}^{2}+u_{3}^{2}\right)-\frac{B_{3}}{2} \\
& \Rightarrow \quad L\left(t, S, E, I, R, u_{1}, u_{2}, u_{3}\right) \geq \min \left(\frac{B_{1}}{2}, \frac{B_{2}}{2}, \frac{B_{3}}{2}\right)\left|\left(u_{1}, u_{2}, u_{3}\right)\right|^{2}-\frac{B_{3}}{2} .
\end{aligned}
$$

Therefore $L\left(t, S, E, I, R, u_{1}, u_{2}, u_{3}\right) \geq d_{1}\left|\left(u_{1}, u_{2}, u_{3}\right)\right|^{\tau}-d_{2}$, where $d_{1}=\min \left(\frac{B_{1}}{2}, \frac{B_{2}}{2}, \frac{B_{3}}{2}\right), d_{2}=$ $\frac{B_{3}}{2}$, and $\tau=2$.

\subsection{Characterization of the optimal controls}

Necessary conditions that optimal solutions need to satisfy are obtained from Pontryagin's maximum principle. This principle converts Eqs. (24)-(26) into a problem of minimizing pointwise a Hamiltonian $(\mathrm{H})$ with respect to $u_{1}, u_{2}$, and $u_{3}$ given by

$$
H\left(t, S, E, I, R, u_{1}, u_{2}, u_{3}, \lambda_{1}, \lambda_{2}, \lambda_{3}, \lambda_{4}\right)=L\left(t, S, E, I, R, u_{1}, u_{2}, u_{3}\right)+\sum_{i=1}^{4} \lambda_{i} f_{i},
$$

where $L\left(t, S, E, I, R, u_{1}, u_{2}, u_{3}\right)=E+I+\frac{B_{1}}{2} u_{1}^{2}+\frac{B_{2}}{2} u_{2}^{2}+\frac{B_{3}}{2} u_{3}^{2}, f_{i}, i=1,2,3,4$, are the right-hand side components of model (24), and $\lambda_{i}, i=1,2,3,4$, are the adjoint or costate variables associated with $S, E, I$, and $R$. Then, applying the Pontryagin's maximum principle, we obtain the following result. 
Theorem 4.2 There exist optimal controls $u_{1}^{*}, u_{2}^{*}$, and $u_{3}^{*}$ and the corresponding state solutions $S^{*}, E^{*}, I^{*}$, and $R^{*}$ that minimize $J\left(u_{1}, u_{2}, u_{3}\right)$ over $U$, and therefore there exist adjoint variables $\lambda_{1}, \lambda_{2}, \lambda_{3}$, and $\lambda_{4}$ such that

$$
\left\{\begin{array}{l}
\frac{d \lambda_{1}}{d t}=\lambda_{1}\left(\mu+\left(1-u_{1}^{*}\right) \frac{\beta I^{*}}{N}\right)-\lambda_{2}\left(\left(1-u_{1}^{*}\right) \frac{\beta I^{*}}{N}\right) \\
\frac{d \lambda_{2}}{d t}=-1+\lambda_{2}\left(\mu+k+\left(1+u_{2}^{*}\right) \alpha\right)-\lambda_{3} k-\lambda_{4}\left(1+u_{2}^{*}\right) \alpha \\
\frac{d \lambda_{3}}{d t}=-1+\lambda_{1}\left(\left(1-u_{1}^{*}\right) \frac{\beta S^{*}}{N}\right)-\lambda_{2}\left(\left(1-u_{1}^{*}\right) \frac{\beta S^{*}}{N}\right)+\lambda_{3}\left(\mu+\gamma+u_{3}^{*}\right)-\lambda_{4}\left(\gamma+u_{3}^{*}\right), \\
\frac{d \lambda_{4}}{d t}=\mu \lambda_{4}
\end{array}\right.
$$

with transversality conditions

$$
\lambda_{i}\left(t_{f}\right)=0, \quad i=1,2,3,4 \text {. }
$$

Furthermore, the characterization of the optimal controls is given by

$$
\begin{aligned}
& u_{1}^{*}(t)=\min \left\{\max \left\{0, \frac{\left(\lambda_{2}-\lambda_{1}\right) \beta S^{*} I^{*} / N}{B_{1}}\right\}, u_{1 \max }\right\}, \\
& u_{2}^{*}(t)=\min \left\{\max \left\{0, \frac{\left(\lambda_{2}-\lambda_{4}\right) \alpha E^{*}}{B_{2}}\right\}, u_{2 \max }\right\}, \\
& u_{3}^{*}(t)=\min \left\{\max \left\{0, \frac{\left(\lambda_{3}-\lambda_{4}\right) I^{*}}{B_{3}}\right\}, u_{3 \max }\right\} .
\end{aligned}
$$

Proof The adjoint system is obtained by taking the negative partial derivatives of $\mathrm{H}$ with respect to each state variable [13], evaluated at optimal controls and corresponding state variables as follows:

$$
\begin{aligned}
& \frac{d \lambda_{1}}{d t}=\frac{-\partial H}{\partial S}=\lambda_{1}\left(\mu+\left(1-u_{1}^{*}\right) \frac{\beta I^{*}}{N}\right)-\lambda_{2}\left(\left(1-u_{1}^{*}\right) \frac{\beta I^{*}}{N}\right), \quad \lambda_{1}\left(t_{f}\right)=0, \\
& \frac{d \lambda_{2}}{d t}=\frac{-\partial H}{\partial E}=-1+\lambda_{2}\left(\mu+k+\left(1+u_{2}^{*}\right) \alpha\right)-\lambda_{3} k-\lambda_{4}\left(1+u_{2}^{*}\right) \alpha, \quad \lambda_{2}\left(t_{f}\right)=0, \\
& \frac{d \lambda_{3}}{d t}=\frac{-\partial H}{\partial I}=-1+\lambda_{1}\left(\left(1-u_{1}^{*}\right) \frac{\beta S^{*}}{N}\right)-\lambda_{2}\left(\left(1-u_{1}^{*}\right) \frac{\beta S^{*}}{N}\right)+\lambda_{3}\left(\mu+\gamma+u_{3}^{*}\right) \\
& \quad-\lambda_{4}\left(\gamma+u_{3}^{*}\right), \quad \lambda_{3}\left(t_{f}\right)=0, \\
& \frac{d \lambda_{4}}{d t}=\frac{-\partial H}{\partial R}=\mu \lambda_{4}, \quad \lambda_{4}\left(t_{f}\right)=0 .
\end{aligned}
$$

Also, for characterization of the optimal controls, we first obtain the optimality conditions by taking the partial derivatives of $\mathrm{H}$ with respect to each control $u_{i}, i=1,2$, 3 , and setting them to zero, that is,

$$
\begin{aligned}
& \frac{\partial H}{\partial u_{1}}=B_{1} u_{1}+\frac{\lambda_{1} \beta S I}{N}-\frac{\lambda_{2} \beta S I}{N}=0 \Rightarrow u_{1}^{*}(t)=\frac{\left(\lambda_{2}-\lambda_{1}\right) \beta S I / N}{B_{1}} \\
& \frac{\partial H}{\partial u_{2}}=B_{2} u_{2}-\lambda_{2} \alpha E+\lambda_{4} \alpha E=0 \Rightarrow u_{2}^{*}(t)=\frac{\left(\lambda_{2}-\lambda_{4}\right) \alpha E}{B_{2}} \\
& \frac{\partial H}{\partial u_{3}}=B_{3} u_{3}-\lambda_{3} I+\lambda_{4} I=0 \Rightarrow u_{3}^{*}(t)=\frac{\left(\lambda_{3}-\lambda_{4}\right) I}{B_{3}} .
\end{aligned}
$$


Then, using the property of the control bounds $0 \leq u_{i} \leq u_{i \max }, i=1,2,3$, the controls are given as

$$
\begin{aligned}
& u_{1}^{*}= \begin{cases}0 & \text { if }\left(\lambda_{2}-1\right) \beta S I / N<0, \\
u_{1}^{*} & \text { if } 0 \leq \frac{\left(\lambda_{2}-\lambda_{1}\right) \beta S I}{N} \leq B_{1} u_{1 \max } \\
u_{1 \max } & \text { if } \frac{\left(\lambda_{2}-\lambda_{1}\right) \beta S I}{N}>B_{1} u_{1 \max }\end{cases} \\
& u_{2}^{*}= \begin{cases}0 & \text { if }\left(\lambda_{2}-\lambda_{4}\right) \alpha E<0 \\
u_{2}^{*} & \text { if } 0 \leq\left(\lambda_{2}-\lambda_{4}\right) \alpha E \leq B_{2} u_{2 \max } \\
u_{2 \max } & \text { if }\left(\lambda_{2}-\lambda_{4}\right) \alpha E>B_{2} u_{2 \max }\end{cases}
\end{aligned}
$$

and

$$
u_{3}^{*}= \begin{cases}0 & \text { if }\left(\lambda_{3}-\lambda_{4}\right) I<0 \\ u_{3}^{*} & \text { if } 0 \leq\left(\lambda_{3}-\lambda_{4}\right) I \leq B_{3} u_{3 \max } \\ u_{3 \max } & \text { if }\left(\lambda_{3}-\lambda_{4}\right) I>B_{3} u_{3 \max } .\end{cases}
$$

This can be written in compact form as follows:

$$
\begin{aligned}
& u_{1}^{*}(t)=\min \left\{\max \left\{0, \frac{\left(\lambda_{2}^{*}-\lambda_{1}^{*}\right) \beta S^{*} I^{*} / N}{B_{1}}\right\}, 1\right\}, \\
& u_{2}^{*}(t)=\min \left\{\max \left\{0, \frac{\left(\lambda_{2}^{*}-\lambda_{4}^{*}\right) \alpha E^{*}}{B_{2}}\right\}, 1\right\}, \\
& u_{3}^{*}(t)=\min \left\{\max \left\{0, \frac{\left(\lambda_{3}^{*}-\lambda_{4}^{*}\right) I^{*}}{B_{3}}\right\}, 1\right\} .
\end{aligned}
$$

We point out that the optimal controls and the corresponding state solutions are found by solving the following optimality system, which consists of the state system (24) with its initial conditions coupled with the adjoint system (28) and its transversality conditions (29) together with the characterization of the optimal controls (30):

$$
\left\{\begin{array}{l}
\frac{d S}{d t}=\mu N-\mu S-\left(1-u_{1}^{*}\right) \frac{\beta S I}{N}, \\
\frac{d E}{d t}=\left(1-u_{1}^{*}\right) \frac{\beta S I}{N}-\left(\mu+k+\left(1+u_{2}^{*}\right) \alpha\right) E, \\
\frac{d I}{d t}=k E-\left(\mu+\gamma+u_{3}^{*}\right) I \\
\frac{d R}{d t}=\left(1+u_{2}^{*}\right) \alpha E+\left(\gamma+u_{3}^{*}\right) I-\mu R, \\
\frac{d \lambda_{1}}{d t}=\lambda_{1}\left(\mu+\left(1-u_{1}^{*}\right) \frac{\beta I}{N}\right)-\lambda_{2}\left(\left(1-u_{1}^{*}\right) \frac{\beta I}{N}\right), \\
\frac{d \lambda_{2}}{d t}=-1+\lambda_{2}\left(\mu+k+\left(1+u_{2}^{*}\right) \alpha\right)-\lambda_{3} k-\lambda_{4}\left(1+u_{2}^{*}\right) \alpha, \\
\frac{d \lambda_{3}}{d t}=-1+\lambda_{1}\left(\left(1-u_{1}^{*}\right) \frac{\beta S}{N}\right)-\lambda_{2}\left(\left(1-u_{1}^{*}\right) \frac{\beta S}{N}\right)+\lambda_{3}\left(\mu+\gamma+u_{3}^{*}\right)-\lambda_{4}\left(\gamma+u_{3}^{*}\right), \\
\frac{d \lambda_{4}}{d t}=\mu \lambda_{4}, \quad \quad \quad \quad \quad \quad I(0)=I_{0}, \\
S(0)=S_{0}, \quad \quad R(0)=E_{0}, \\
\lambda_{i}\left(t_{f}\right)=0, \quad i=1,2,3,4 .
\end{array}\right.
$$

\subsection{Uniqueness of the optimality system}

Since the state and adjoint variables are bounded (because the adjoint system (28) is also linear in $\lambda_{i}, i=1,2,3,4$, as the state system) and satisfy the Lipschitz condition rela- 


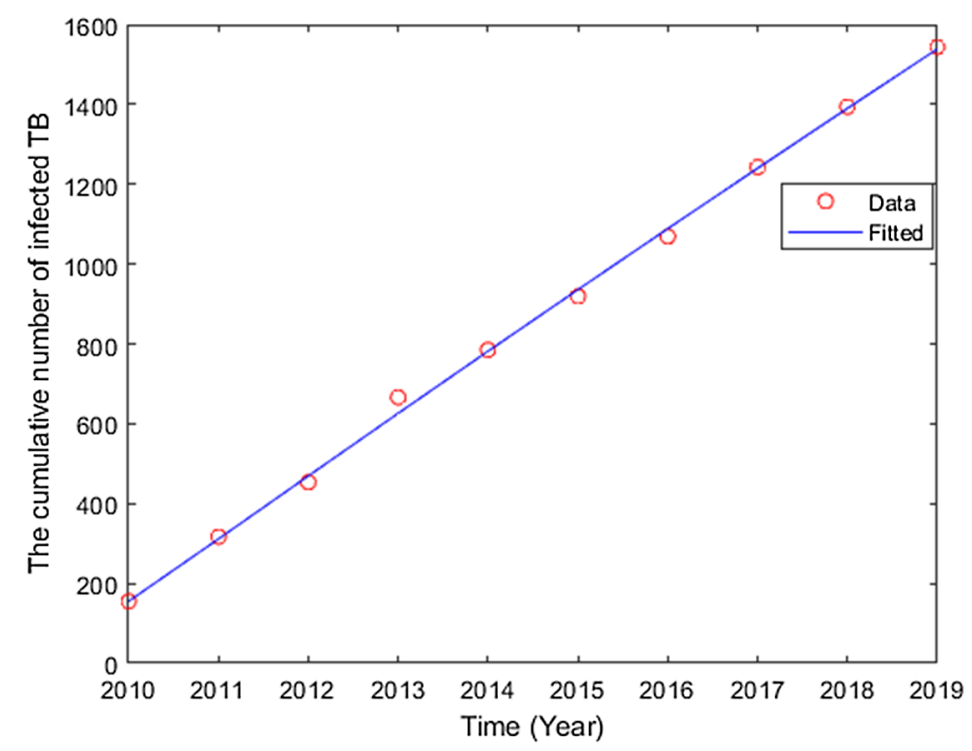

Figure 2 Simulation results for the cumulative number of people infected with TB

tive to their state variables, it is possible to show the uniqueness of the optimality system.

Theorem 4.3 If the state and adjoint variables are bounded and satisfy the Lipschitz condition relative to their state variables with constant $M>0$, then for the sufficiently small final time $t_{f}$, the solutions of system (31) are unique (for proof, see [14]).

\section{Numerical results and discussions}

In this section, we first estimate the model parameters. Second, we analyze the stability of the equilibrium points. Then we investigate the effect of the controls in reducing the infected population with TB under different strategies.

\subsection{Estimation of model parameters}

Since some parameter values such as demographic data are well known from the literature, we have estimated unknown parameters based on the data obtained from the health office of the Haramaya district and Haramaya hospital to keep the model more realistic. The natural mortality $\mu$ is postulated to be equal to the inverse of the life expectancy at birth, which is now about 65.5 years in Ethiopia [22]. Therefore the natural death rate $\mu$ in Ethiopia is $\frac{1}{65.5}=0.015267$ per year. The mean infectious period $\frac{1}{\gamma}$ is estimated in the range of $0.5-2$ years. Here we choose $\gamma=\frac{0.5+2}{2}=1.25$ year $^{-1}$. The parameter $\beta$ is in the range of $10-15$ year $^{-1}$ by the assumption that a person with active TB infects an average of $10-15$ other people every year [18]. So, we have taken $\beta=10$ year $^{-1}$. The parameters $\mathrm{k}$ and $\alpha$, which cannot be obtained by the observed data or references, are estimated using the least-squares data fitting method with the help of a MATLAB tool fminsearch, which is part of the optimization toolbox. This is done by fitting model (1) to the observed data of active TB incidence in 2010-2019 ensuring that the minimum error occurred whilst getting the best fits. The fitted curve to the observed data is shown in Fig. 2 and the estimated values of $k$ and $\alpha$ are presented in Table 2. 
Table 2 Parameter estimates and 95\% Cl calculated from 1000 simulated data sets

\begin{tabular}{lll}
\hline Parameter & Estimate & $95 \% \mathrm{Cl}$ \\
\hline $\mathrm{k}$ & 0.0444 & $(0.0392,0.0501)$ \\
$\alpha$ & 0.2325 & $(0.1689,0.3077)$ \\
\hline
\end{tabular}

To quantify parameter uncertainty and construct confidence intervals (CI), we rely on the parametric bootstrapping method. Bootstrapping is a statistical method for assigning accuracy measures to the sample estimates [6]. In this method, multiple observations are repeatedly sampled from the best-fit model to quantify parameter uncertainty by assuming that the time series follow a Poisson distribution centered on the mean at the time points.

In addition to the parameters, we take the following as an initial population of the district relating to the year 2010, and for numerical results, we divide the total population $N=$ 361,000 , as follows. We assume that $80 \%$ of the population are susceptible [18], whereas the number $I_{0}$ of infectious individuals in 2010 was estimated by the number of smear positive patients obtained from the health office of the Haramaya district and Haramaya hospital. So, with this assumption, we have the following initial data for the simulation purpose: $\left(S_{0}, E_{0}, I_{0}, R_{0}\right)=(288,800,3455,155,68,590)$.

\subsection{Stability analysis of equilibrium points}

Based on the above real data, we found from Eq. (12) that $R_{0}=1.2011>1$. This in principle shows that TB does spread in the community of the district. Since $R_{0}>1$, the prevalence of TB will result in an epidemic. This is due to the fact that the rate of transmission is greater than the recovery rate. From Eq. (10), the DFE point is determined to be $E_{1}=(361,000,0,0)$, and from Eq. (11), EE point $E_{2}=\left(\frac{N}{R_{0}}, \frac{\mu N(\mu+\gamma)}{\beta k}\left(R_{0}-1\right), \frac{\mu N}{\beta}\left(R_{0}-1\right)\right)=$ (300557.8, 3158.4, 110.8).

The characteristic equation from (17) becomes

$$
\lambda^{3}+1.5727 \lambda^{2}-0.0506 \lambda-0.0011=0
$$

which shows that the DFE point is unstable by the Routh-Hurwitz criterion. This means that when an individual infected with Mycobacterium TB is present in a susceptible population, it will eventually result in an outbreak of the disease. The characteristic equation from (21) also becomes

$$
\lambda^{3}+1.5758 \lambda^{2}+0.0286 \lambda+0.0011=0,
$$

which shows that the EE point is stable. This means that TB in the study area will persist.

\subsection{Optimal control problem analysis}

In this section, we numerically analyze the impact of control strategies on controlling transmission of the TB disease using the Matlab R2019a program. We solve the optimality system (31), which consists of eight ordinary differential equations from state and adjoint equations, using the forward-backward sweep method developed by Lenhart and Workman [13]. The numerical procedure starts with an initial guess on the control variables. Then, using fourth-order Runge-Kutta scheme, we solve the state equations simultaneously forward in time starting from the initial conditions, and using the state solutions, we 

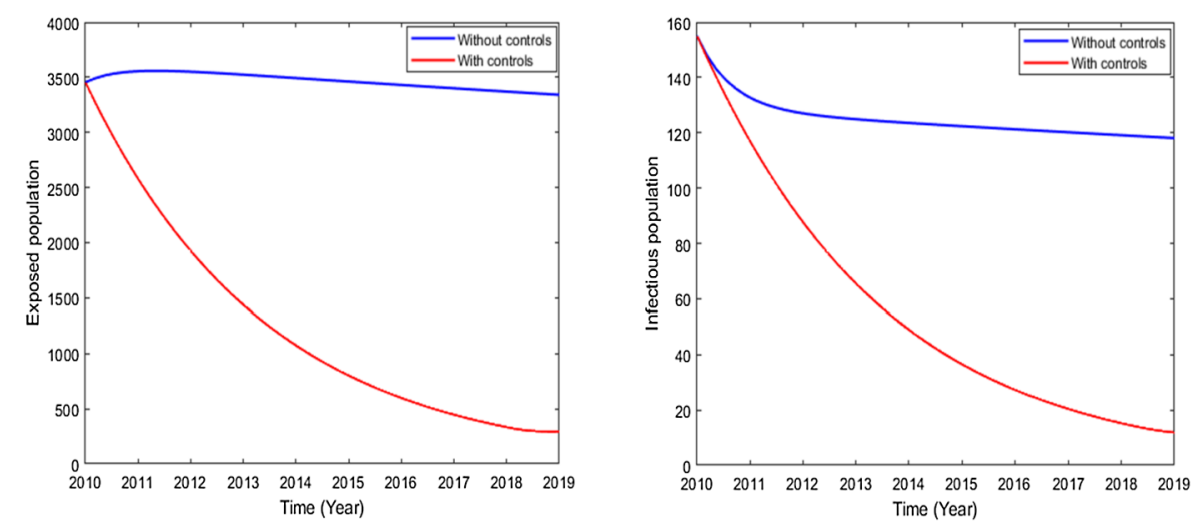

Figure 3 Simulations of the model showing the effect of strategy A

solve the adjoint equations backward in time simultaneously starting from the transversality conditions. The controls are updated by using a convex combination of the previous controls and the characterization values. The we utilize the updated controls to repeat the solutions of state and adjoint systems. This process is repeated until the values of the state, adjoint, and control variables at the previous iteration are very close to those at the present iteration.

To illustrate the numerical results, we consider $B_{1}=B_{2}=B_{3}=100$. The impact of each control strategy on eradication of the TB disease is investigated under the following strategies:

i. Strategy A: Applying distancing $\left(u_{1}\right)$ only.

ii. Strategy B: Applying case finding $\left(u_{2}\right)$ only.

iii. Strategy C: Applying treatment $\left(u_{3}\right)$ only.

iv. Strategy D: Combination of use of distancing $\left(u_{1}\right)$ and case finding $\left(u_{2}\right)$.

v. Strategy E: Combination of use of distancing $\left(u_{1}\right)$ and treatment $\left(u_{3}\right)$.

vi. Strategy F: Combination of use of case finding $\left(u_{2}\right)$ and treatment $\left(u_{3}\right)$.

vii. Strategy G: Combination of use of distancing $\left(u_{1}\right)$, case finding $\left(u_{2}\right)$, and treatment $\left(u_{3}\right)$.

\subsubsection{Strategy A}

Here we only use distancing control $u_{1}$ to optimize the objective functional $J$ in (25). From Fig. 3 we observe that the strategy has a significant effect in reducing the number of exposed and infectious populations when compared to the numbers in the case without controls.

\subsubsection{Strategy B}

Under this strategy, we use the case finding control $u_{2}$ to optimize the objective functional $J$ in (25) while setting other controls to zero. From Fig. 4 we observe that this strategy is effective in reducing the number of exposed and infectious populations.

\subsubsection{Strategy $C$}

In this strategy, we only use treatment control $u_{3}$ to optimize the objective functional $J$ in (25) while setting other controls to zero. From Fig. 5 we observe that the strategy is effective in reducing the number of exposed and infectious populations. 

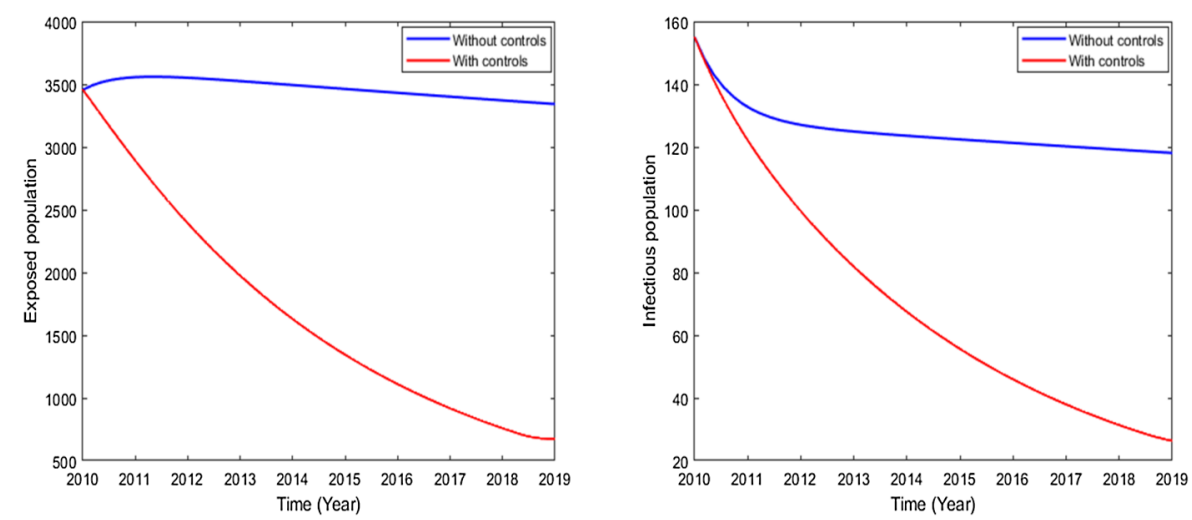

Figure 4 Simulations of the model showing the effect of strategy $B$
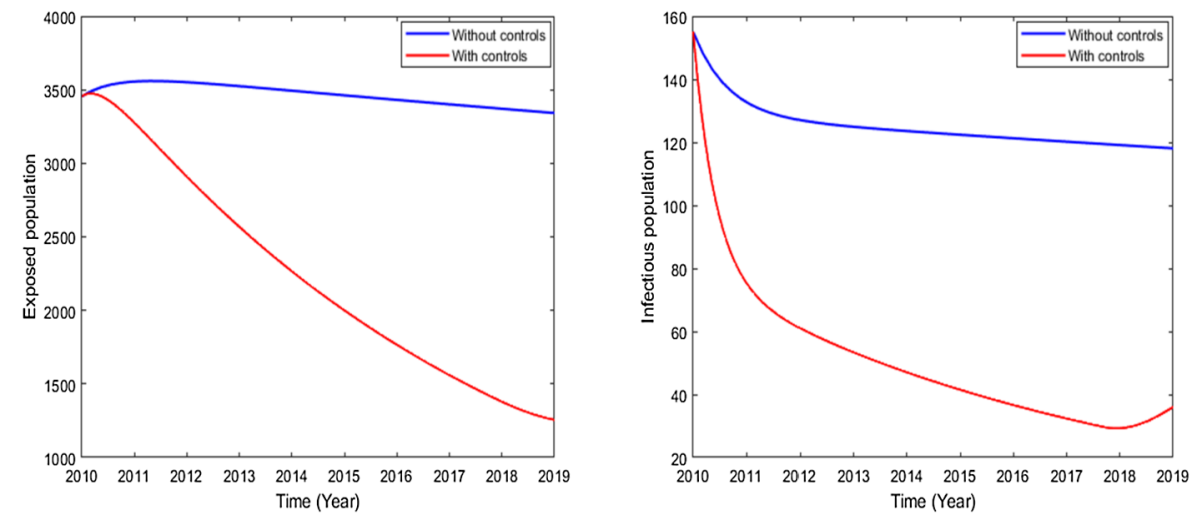

Figure 5 Simulations of the model showing the effect of strategy $C$

From the numerical computation we get that the numbers of infectives with strategies A, B, and C are 306, 701, and 1292, respectively, at final time. In Figs. 6(a), 6(b), and 6(c), the control profiles of strategies $\mathrm{A}, \mathrm{B}$, and $\mathrm{C}$ are displayed, which suggest that controls $u_{1}, u_{2}$, and $u_{3}$ of the respective strategy are at upper bound for period of 8.1, 8.3, and 7.7 years, respectively, before sharply drop to zero at final time. These clearly indicate that the number of infection is reduced by a larger amount in strategy A than in strategy B and C, whereas strategy $\mathrm{C}$ requires less effort than strategies $\mathrm{A}$ and $\mathrm{B}$.

\subsubsection{Strategy D}

The distancing control $u_{1}$ and case finding control $u_{2}$ are applied under this strategy, whereas treatment control $u_{3}$ is set to zero. We observe from Fig. 7 that the number of exposed and infectious populations decrease when compared to the numbers in the case without controls.

\subsubsection{Strategy E}

Here both distancing control $u_{1}$ and treatment control $u_{3}$ are used, whereas case finding control $u_{2}$ is set to zero. We observe from Fig. 8 that the strategy is effective in reducing the number of exposed and infectious populations. 

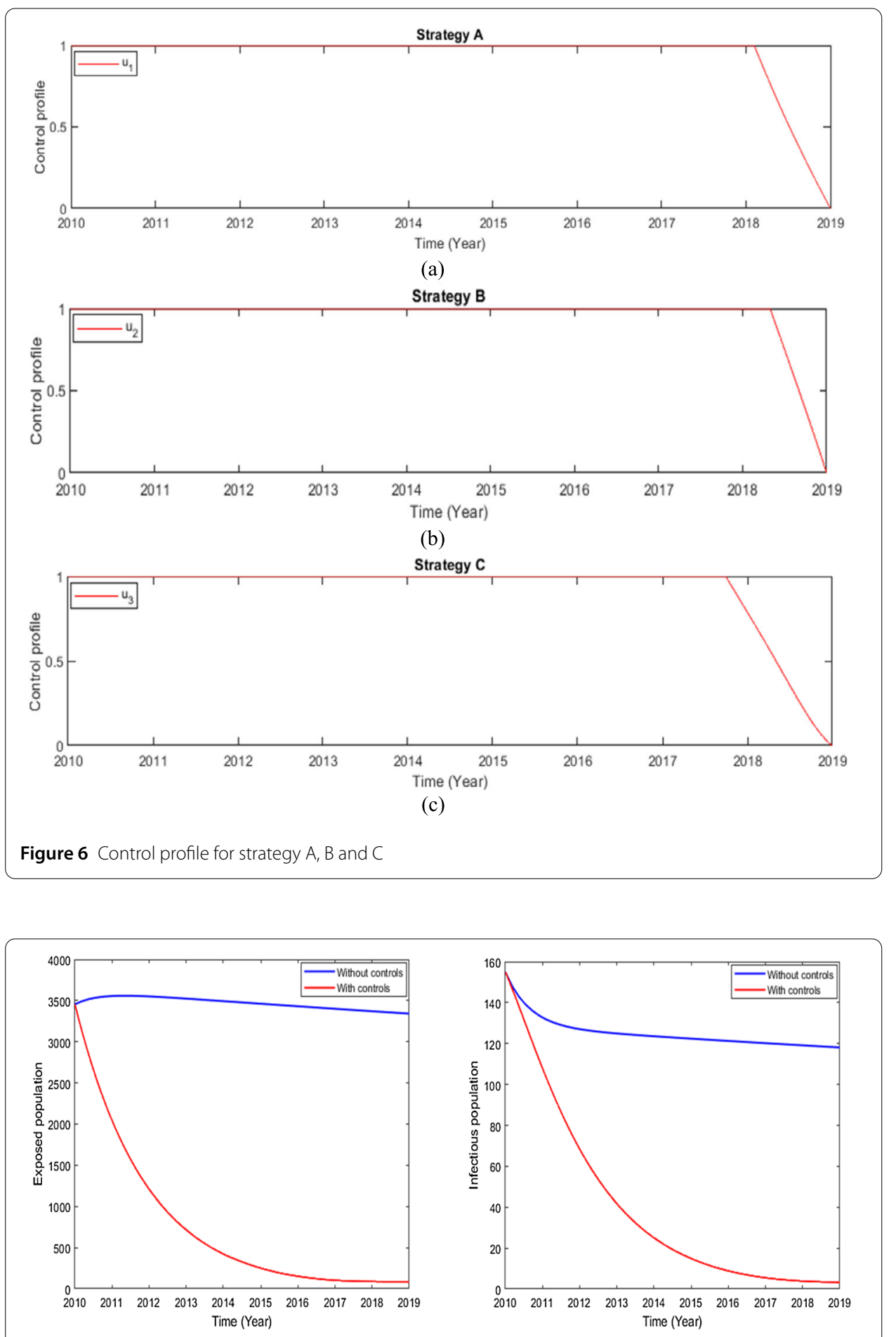

Figure 7 Simulations of the model showing the effect of strategy $D$

\subsubsection{Strategy F}

In this strategy, the objective functional $J$ is optimized using case finding control $u_{2}$ and treatment control $u_{3}$. We observe from Fig. 9 that this strategy shows a significant effect in reducing the number of exposed and infectious populations. 

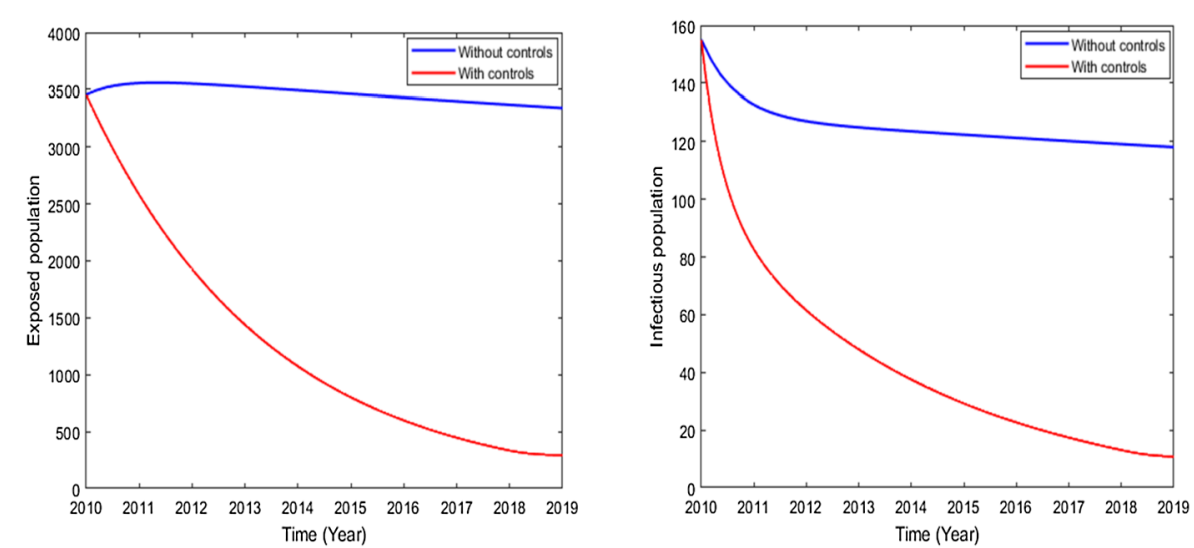

Figure 8 Simulations of the model showing the effect of strategy $E$
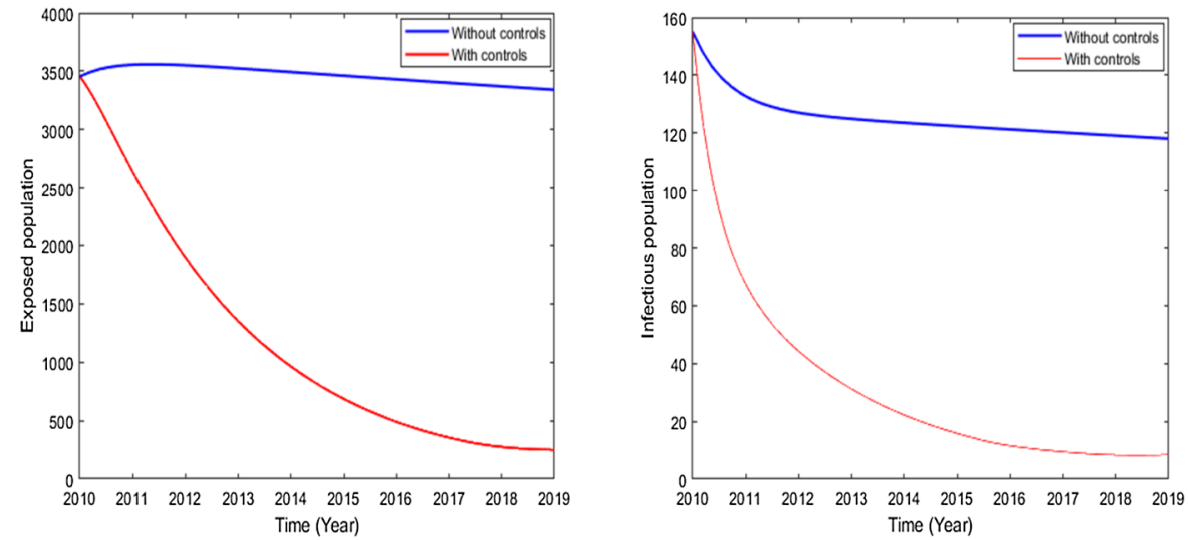

Figure 9 Simulations of the model showing the effect of strategy $F$
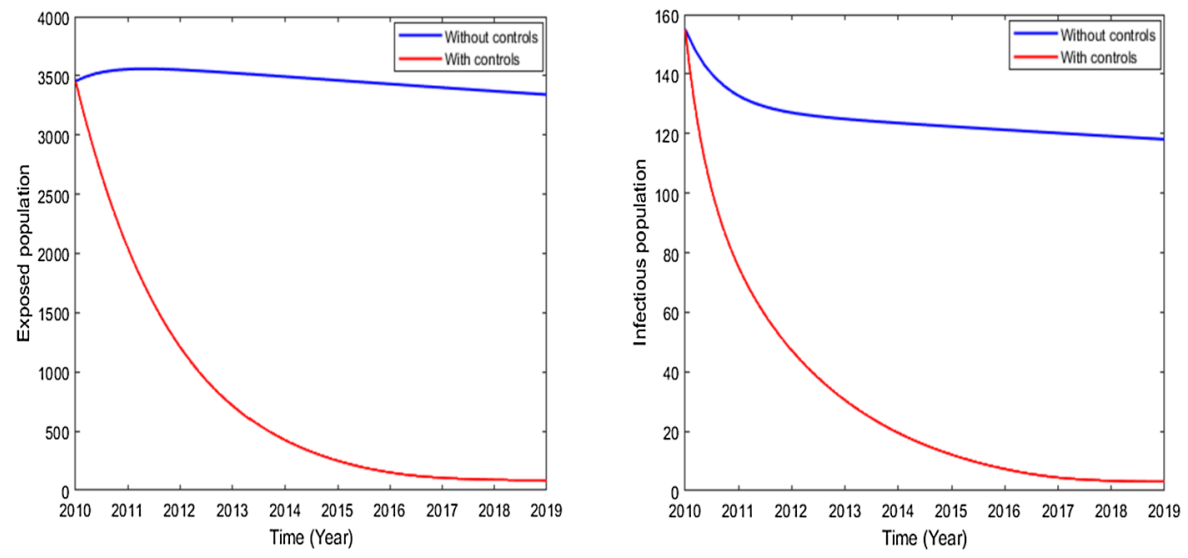

Figure 10 Simulations of the model showing the effect of strategy $G$ 


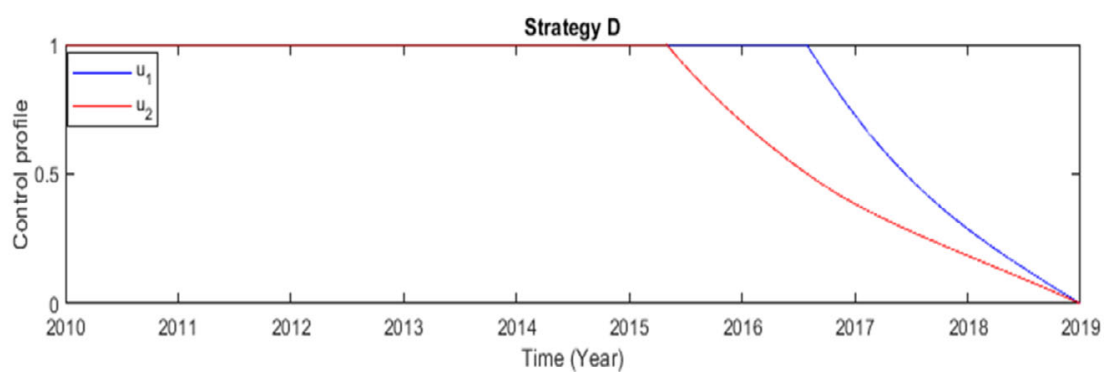

(a)

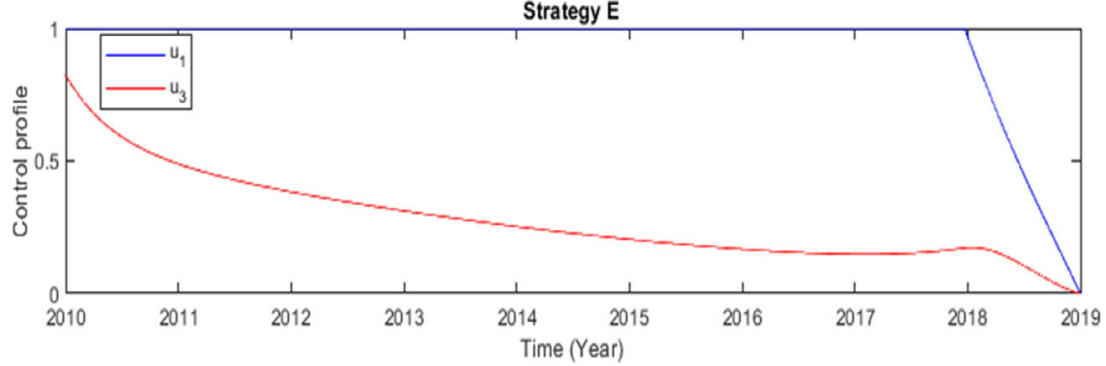

(b)

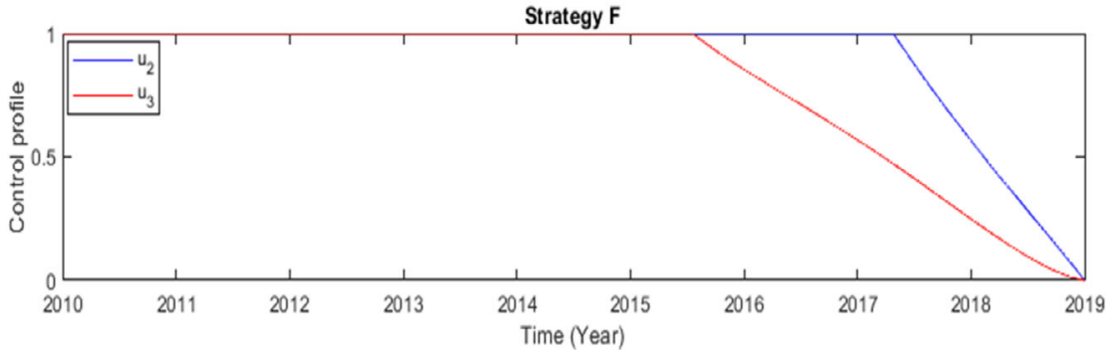

(c)

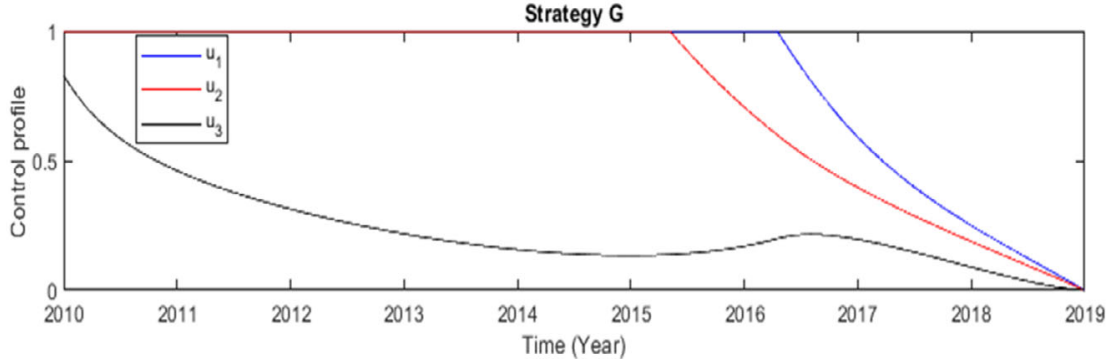

(d)

Figure 11 Control profile for strategy D, E, F, and G

\subsubsection{Strategy $G$}

Here we used all the three control strategies $u_{1}, u_{2}$, and $u_{3}$ to optimize the objective functional $J$. We observe from Fig. 10 that the number of exposed and infectious populations highly decrease when compared to the numbers in the case without controls.

The results from the numerical computations show that the numbers of infectives with strategies D, E, F, and G are 89, 305, 259, and 88, respectively, at final time, which indicates that strategy $\mathrm{G}$ is more effective in reducing the number of infection than other strategies. In Figs. 11(a), 11(b), 11(c), and 11(d) the control profiles of strategy D, E, F, and G are shown, which indicate that strategy $\mathrm{G}$ requires less effort than other strategies. 


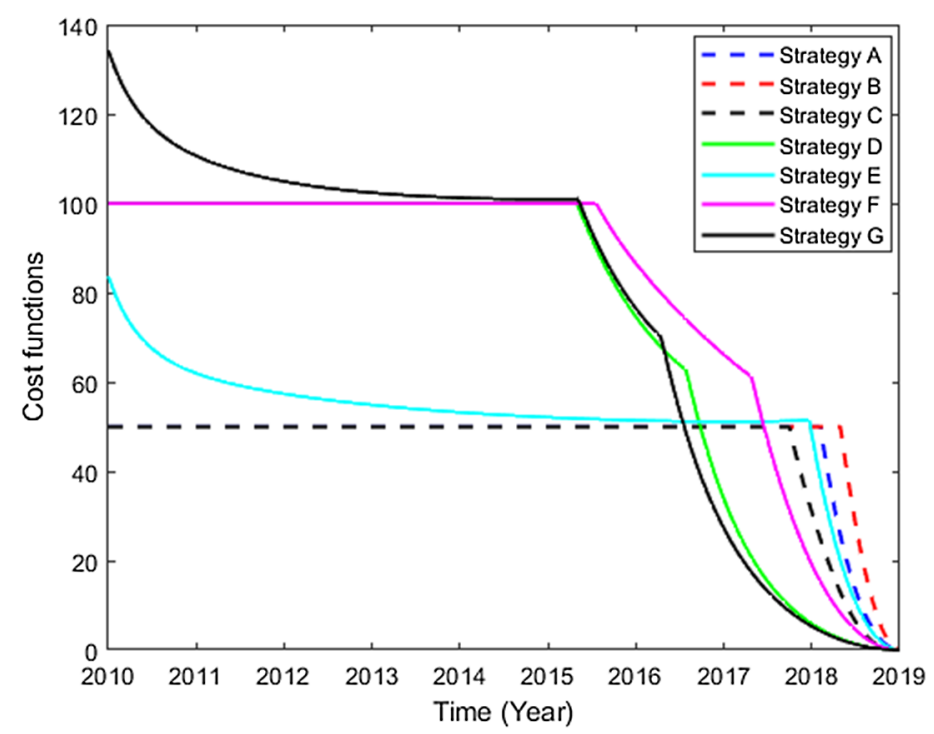

Figure 12 Cost function of the intervention strategies

From the above results, all strategies are effective in reducing the number of exposed and infectious populations when compared with a situation without controls. The strategies with only one control are less effective in reducing the number of exposed and infectious populations when compared to strategies that incorporate more than one control. Also, even if the strategies with only one control are less effective, they are cheaper than the strategies that incorporate more than one control, as indicated in Fig. 12.

\section{Conclusion}

In this paper, we developed a dynamic model for TB transmission in the Haramaya district of Ethiopia based on active-TB incidence data recorded by the district health office and the Haramaya hospital. The model qualitative analysis demonstrates that model solutions are bounded and positive. The basic reproduction number and local stability conditions of the equilibria were determined, and the basic reproduction number was used to perform the sensitivity analysis that identified the role of each parameter used in the model to the spread of TB disease. Three time-dependent control measures such as distancing, case finding through screening of TB exposed individuals, and treatment of TB infectious population were applied to the model so that basic model was extended into an optimal control model. Theoretically, we proved the existence of an optimal control and obtained necessary conditions that optimal solutions need to satisfy using Pontryagin's maximum principle. Numerically, the levels of exposed and infectious populations with and without optimal controls were compared under different intervention strategies to investigate the roles of control measures independently and as combination in eradication of the TB disease. Implementing strategies with only one control is less effective in reducing the number of exposed and infectious populations when compared with strategies that incorporate more than one control. From strategies that incorporate more than one control measure, the strategy with combination of all three control measures is the best strategy in reducing the number of $\mathrm{TB}$ infection with minimum cost of interventions. 


\section{Acknowledgements}

The authors are grateful to Haramaya University for the financial support and health office of the Haramaya district and Haramaya hospital for their assistance in data collection. The authors thank the anonymous referees of the paper for their constructive comments and suggestions.

\section{Funding}

Not applicable

Availability of data and materials

The datasets used during the current study are available from the first author on reasonable request.

\section{Ethics approval and consent to participate}

Not applicable.

\section{Competing interests}

The authors declare that they have no competing interests.

\section{Consent for publication}

Not applicable.

\section{Authors' contributions}

The authors declare that the study was realized in collaboration with equal responsibility. Both authors read and approved the final manuscript.

\section{Publisher's Note}

Springer Nature remains neutral with regard to jurisdictional claims in published maps and institutional affiliations.

Received: 13 November 2020 Accepted: 2 June 2021 Published online: 12 June 2021

\section{References}

1. Abouelkheir, I., Alkama, M., Elkihal, F., Rachik, M.: Optimal control strategy of a tuberculosis epidemic model with drug resistant TB. Int. J. Sci. Res. 4(3), 427-430 (2015)

2. Athithan, S., Ghosh, M.: Optimal control of TB with case detection and treatment. World J. Model. Simul. 11(2), 111-122 (2015)

3. Berhe, H.W., Makinde, O.D., Theuri, D.M.: Parameter estimation and sensitivity analysis of dysentery diarrhea epidemic model. J. Appl. Math. 2019, Article ID 8465747 (2019). https://doi.org/10.1155/2019/8465747

4. Castillo-Chavez, C., Feng, Z., Huang, W.: On the computation of $R_{0}$ and its role on global stability. In: Mathematical Approaches for Emerging and Reemerging Infectious Diseases, pp. 229-250. Springer, New York (2002). https://doi.org/10.1007/978-1-4613-0065-6

5. Castillo-Chavez, C., Song, B.: Dynamical models of TB and their applications. Math. Biosci. Eng. 1(2), 361-404 (2004)

6. Choi, S., Jung, E.: Optimal TB prevention and control strategy from a mathematical model based on real data. Bull. Math. Biol. 76, 1566-1589 (2014). https://doi.org/10.1007/s11538-014-9962-6

7. Coddington, E.A.: An Introduction to Ordinary Differential Equations. Prentice-Hall, Upper Saddle River (1961)

8. Coddington, E.A., Levinson, N.: Theory of Ordinary Differential Equations. McGraw-Hill, New York (1955)

9. Dontwi, I.K., Obeng-Denteh, W., Andam, E.A., Obiri-Apraku, L.: A mathematical model to predict the prevalence and transmission dynamics of TB in Amansie West district, Ghana. Br. J. Math. Comput. Sci. 4(3), 402-425 (2014)

10. Driessche, P.V., Watmough, J.: Reproduction numbers and sub-threshold endemic equilibria for compartmental models of disease transmission. Math. Biosci. 180(2), 29-48 (2002). https://doi.org/10.1016/S0025-5564(02)00108-6

11. Fleming, W.H., Rishel, R.W.: Deterministic and Stochastic Optimal Control. Springer, New York (1982)

12. Gao, D.P., Huang, N.J.: Optimal control analysis of a tuberculosis model. Appl. Math. Model. 58, 47-64 (2017). https://doi.org/10.1016/j.apm.2017.12.027

13. Lenhart, S., Workman, J.T.: Optimal Control Applied to Biological Models. CRC Press, New York (2007). https://doi.org/10.1201/9781420011418

14. Mlay, G.M., Luboobi, L., Kuznetsov, D., Shahada, F.: Optimal treatment and vaccination control strategies for the dynamics of pulmonary TB. Int. J. Adv. Appl. Math. Mech. 2(3), 196-207 (2015)

15. Okuonghae, D., Omosigho, S.E.: Analysis of a mathematical model for TB: what could be done to increase case detection. J. Theor. Biol. 269(2011), 31-45 (2010)

16. Rodrigues, P., Silva, C.J., Torres, D.F.M.: Cost-effectiveness analysis of optimal control measures for TB. Soc. Math. Biol. (2014). https://doi.org/10.1007/s11538-014-0028-6

17. Shah, N.H., Gupta, J.: Mathematical modelling of pulmonary and extra-pulmonary TB. Int. J. Math. Trends Technol. 4(9), 158-162 (2013)

18. Sintayehu, S.: Modelling the transmission of drug resistant TB in Ethiopia. MSc Thesis, University of Addis Ababa (2013)

19. Ugwa, K.A., Agwu, I.A., Agbanyim, A.N.: Mathematical analysis of the endemic equilibrium of the transmission dynamics of TB. Int. J. Sci. Technol. Res. 2(2), 263-269 (2013)

20. Waaler, H., Geser, A., Andersen, S.: The use of mathematical models in the study of the epidemiology of TB. Am. J. Public Health Nation's Health 52(6), 1002-1013 (1962). https://doi.org/10.2105/ajph.52.6.1002

21. Whang, S., Choi, S., Jung, E.: A dynamic model for TB transmission and optimal treatment strategies in South Korea. J. Theor. Biol. 279(1), 120-131 (2011)

22. World Health Organization: Organization, Global TB Report. World Health Organization, Geneva (2018) 
23. World Health Organization: Organization, Ethiopia's Effort in Fighting Against TB. World Health Organization, Geneva (2019)

24. Zhang, J., Li, Y., Zhang, X.: Mathematical modelling of TB data of China. J. Theor. Biol. 365(2015), 159-163 (2014)

25. Zhao, Y., Li, M.T., Yuan, S.: Analysis of transmission and control of TB in Mainland China, 2005-2016, based on the age-structure mathematical model. Int. J. Environ. Res. Public Health 14(10), 1192 (2017). https://doi.org/10.3390/ijerph14101192

Submit your manuscript to a SpringerOpen ${ }^{\circ}$ journal and benefit from:

- Convenient online submission

- Rigorous peer review

Open access: articles freely available online

- High visibility within the field

Retaining the copyright to your article

Submit your next manuscript at $\gg$ springeropen.com 